\title{
Wearable RFID-Enabled Sensor Nodes for Biomedical Applications
}

\author{
Li Yang, Rushi Vyas, Amin Rida, Jonathan Pan, and Manos M. Tentzeris \\ Georgia Electronic Design Center, School of Electrical and Computer Engineering, \\ Georgia Institute of Technology, Atlanta, GA 30332-0250, USA \\ liyang@ece.gatech.edu
}

\begin{abstract}
A wearable RFID-enabled sensor node for continuous biomedical monitoring is investigated in this paper. Dielectric characterization of fabric substrates, inkjet-printing of conductive nano-particle silver ink, design of RFID antennas and integration of sensor active and passive devices were discussed in this paper. Preliminary experiments show that the RFID-enabled sensor node could be effective for biomedical applications.
\end{abstract}

\section{Introduction}

The growing demand for economic, low power, conformal and durable wireless nodes with sensing capabilities is driven by applications such as: item-level tracking of temperature and humidity, pharmaceutical logistics, transport and storage of medical products and bio-sensing. The major challenge in this type of applications is the need for low-cost eco-friendly wireless sensor nodes that can be easily implemented out in different environments. Radio Frequency Identification (RFID) is a low-cost compact wireless technology, which is not limited by Line of Sight requirements to communicate with an interrogator and convey information concerning the location and the status of an item, that has been already applied to inventory tracking and management, retail level management, access control, animal tracking, medical anticounterfeiting, security, and electronic toll collection. Lately, RFID technology has started getting used into individual use contexts as well. Wearable RFID-enabled sensor is such a branch attracting strong interest and will soon become another fast growing field in application-oriented research.

Flexible, reliable and compact wearable RFID-enabled sensor nodes are required in order to establish and maintain a fast and secured body-area network. To realize optimized designs, it is necessary to determine the influence of substrate on the electromagnetic performance of the sensor node, especially the antenna part. Material characterization with resonator methods will be a must to include the consideration of effects of the textiles in the design procedure. In the next step, a UHF RFID tag will be optimized, deploying inkjetprinting as a fast direct-write solution to fabricate the RFID antenna directly on flexible eco-friendly organic materials. The direct-write technology also provides the feasibility to have all-printed wearable RFID tag integrated with sensors and power sources. A suggested outline of RFID/sensor integration will be illustrated.

The wearable RFID-enabled sensor node can potentially find broad usage in real-time monitoring and medical monitoring applications. In this integrated module, the sensor part will detect the condition of the patient, such as body temperature, and transmit the signal out through the use of the RFID section. The readers implemented over the hospital will gather the information transmitted by the RFID, and trigger the alarm to notify the doctors if some severe condition information is detected. Therefore, real-time biomedical monitoring can be realized with the aid from RFID without adding more labor force. In addition, extra information, such as the recommended patient's drug dosage could be included also in the RFID module of the RFID-enabled sensor node to minimize the risk of overdose or wrong treatment. A similar methodology can be extended to the development of biosensors and wireless body area networks (WBAN) [1], as shown in the Fig. 1.

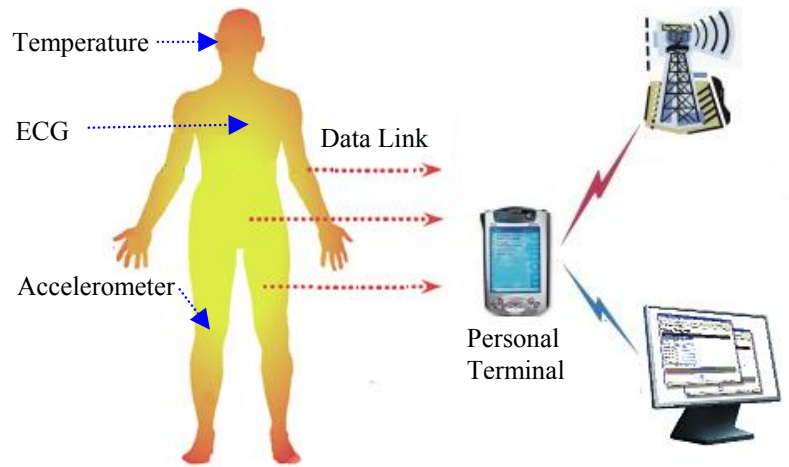

Fig. 1. Conceptual diagram of wireless body area networks (WBAN).

\section{Dielectric Characterization}

Among the critical needs for the selection of the right type of textile for electronics applications are the surface planarity, water-repelling, lamination capability for 3D module development, adhesion, and co-processability with low-cost manufacturing. RF characterization of textile substrate becomes a necessary step for the qualification of the material for a wide range of frequency domain applications. The knowledge of the dielectric properties such as dielectric constant $\left(\varepsilon_{\mathrm{r}}\right)$ and loss tangent $(\tan \delta)$ become necessary for the design of any high frequency structure such as RFID antennas on the textile substrate and more importantly if it is to be embedded inside the substrate. Precise methods for highfrequency dielectric characterization include microstrip ring resonators, parallel plate resonators, and cavity resonators [2].

In order to measure the dielectric constant $\left(\varepsilon_{\mathrm{r}}\right)$ and loss tangent $(\tan \delta)$ of fabrics up to $2 \mathrm{GHz}$, a microstrip ring resonator structure was designed, the configuration diagram is shown in Fig. 2. A calibration method namely throughreflect-lines (TRL) was utilized to de-embed the effect of the feeding lines. It is to be noted that $\tan \delta$ extraction using the microstrip ring resonator approach requires reliable theoretical equations for the estimation of the conductor losses [3]. 

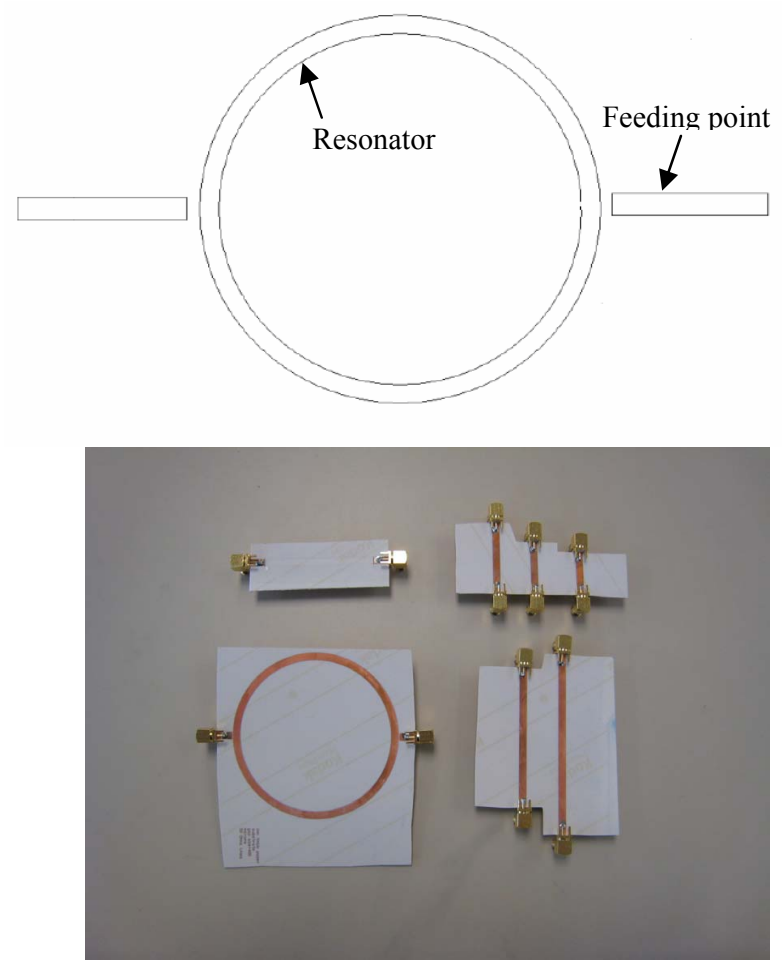

Fig. 2. Schematic layout and photograph of fabricated microstrip ring resonators and TRL lines bonded to SMA connectors.

The ring resonator produces Insertion Loss (S21) results with periodic frequency resonances. In this method, $\varepsilon_{\mathrm{r}}$, can be extracted from the location of the resonances of a given radius ring resonator while tan $\delta$ is extracted from the quality factor (Q) of the resonance peaks along with the theoretical calculations of the conductor losses. Typical SMA coaxial connectors were used to feed the ring resonator structure. TRL calibration was performed to de-embed the input and output microstrip feeding lines effects and eliminate any impedance mismatch.

\section{Conductive Inkjet-printing}

Inkjet-printing is a direct-write technology by which the design pattern is transferred directly to the substrate, and there is no requirement of masks contrary to the widely used traditional etching techniques [4]. In addition, unlike etching which is a subtractive method by removing unwanted metal from the substrate surface and which also uses chemicals such as the etchants throughout the fabrication process, inkjetprinting jets the single ink droplet from the nozzle to the desired position, therefore no waste is created, resulting in an economical fabrication solution. This aspect, together with the fact that the chemicals necessary for etching are eliminated, makes this approach environmentally friendly as well. The printer and print-head are shown in Fig. 3.

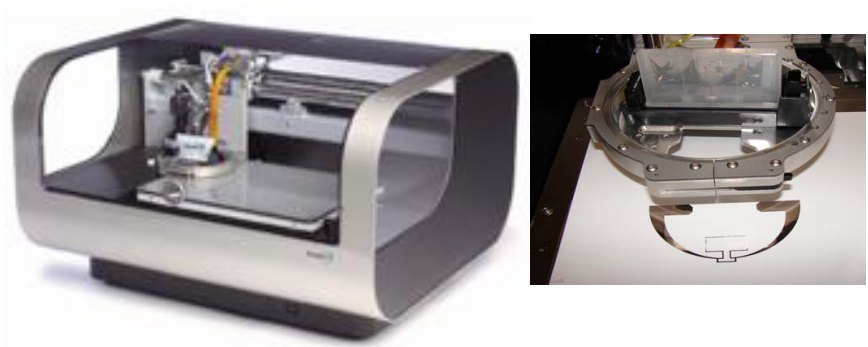

Fig. 3. Material printer DMP-2800 and the print-head DMC11610 .

A photo taken by a fiducial camera of the Material Printer DMP-2800 of an edge section (hence showing the organic substrate to the left of the ink) of a printed transmission line is shown in Fig. 4. The total view width is $1.628 \mathrm{~mm}$ while the total height view is $1.221 \mathrm{~mm}$ with resolution of 2.54 microns.

Curing the conductive particles, such as the nano-silver particles utilized in this effort, has to occur before any operation of the ink as a good conductor. Curing at temperature high temperatures is desirable; however, other methods such as UV or photonic curing may be used, which takes up only few seconds. The inkjet printing approach is very repeatable, allows for features down to $20 \mu \mathrm{m}$ and can be easily utilized for other passive structures, such as filters, baluns in single or multilayer (multi-sheet) configurations.

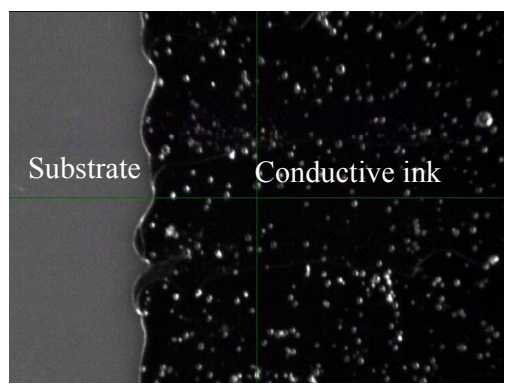

Fig. 4. Photograph of conductive ink on organic substrate with a resolution of 2.54 microns.

\section{Inkjet-printed RFID Antennas}

After the electrical characterization of dielectric constant $\left(\varepsilon_{\mathrm{r}}\right)$ and loss tangent $(\tan \delta)$ of textile over the frequency range has been achieved, the dielectric characterization results have been incorporated in the design of the RFID antennas.

The developing of a passive RFID tag was demonstrated in this section. Passive tags transform energy from an RFID reader antenna to power up the IC and communicate with the RFID reader antenna through backscattering. The main challenge for this application was the miniaturization of the passive tags in the UHF RFID frequency range at which the typical free space wavelength is around $30 \mathrm{~cm}$. Another challenge was the matching of passive RFID antennas to the impedance of the IC, which commonly exhibits highly 
capacitive behaviour, for optimum range and power flow in/out of the passive tag while communicating with the reader.

The design of a half-wavelength tapered-width U-shaped antenna is shown in Fig. 5. The two stubs, namely inductive and shorting stubs are responsible for the matching of the antenna terminals to the IC impedance of value 16-j350 Ohms, which is expected to have a flat response over the UHF RFID frequency band. The resistive stub primarily matched the radiating body's total input impedance to the resistive part of the IC while the inductive stub primarily matches it to the reactance part of the IC. The tapered width of the two arms offers an increased bandwidth. The dimensions of this antenna are $8.2 \mathrm{~cm} \times 4.5 \mathrm{~cm}$.

Introducing stubs is very effective in matching to, theoretically, any arbitrarily IC impedance. The target IC impedance used in this design was Philips EPC 1.19 Gen 2 RFID ASIC IC which exhibits a stable impedance behavior of $16-\mathrm{j} 350 \Omega$ over the frequency $902 \mathrm{MHz}-928 \mathrm{MHz}$. Return Loss plot is shown in Fig. 6 with a bandwidth of $905 \mathrm{MHz}-$ $925 \mathrm{MHz}$ defined by a value of $\mathrm{RL}<-10 \mathrm{~dB}$, with radiation efficiency of $92 \%$. The radiation pattern is shown in Fig. 7.

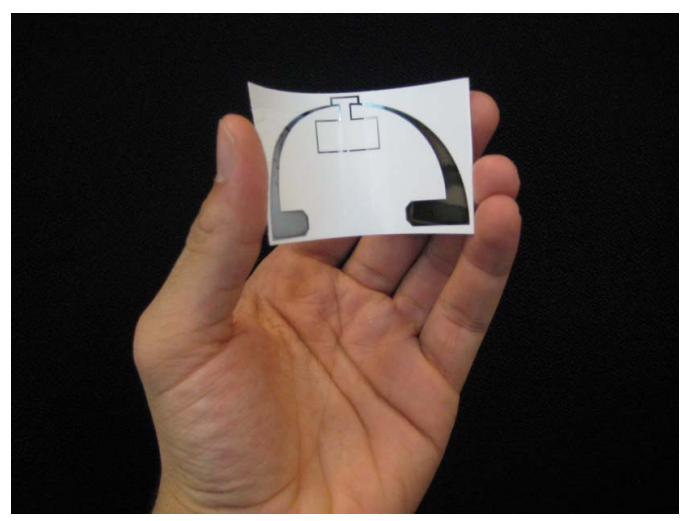

Fig. 5. Photograph of the U-shaped Antenna showing flexibility. Two matching stubs can also be seen.

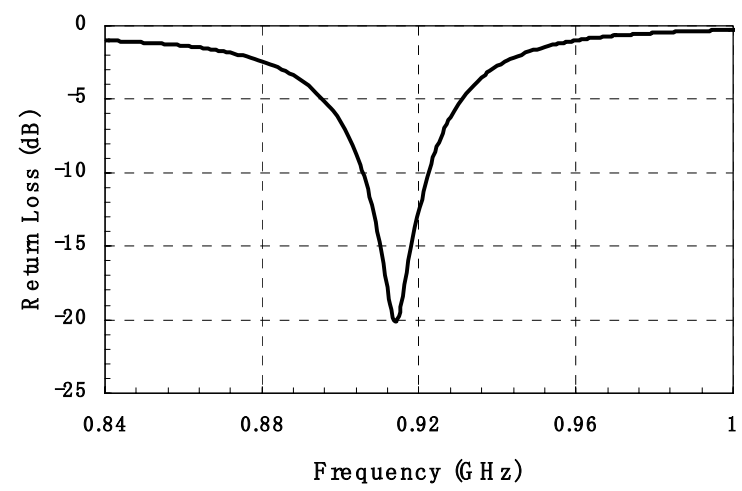

Fig. 6. Return Loss of the U-shaped Antenna showing bandwidth.

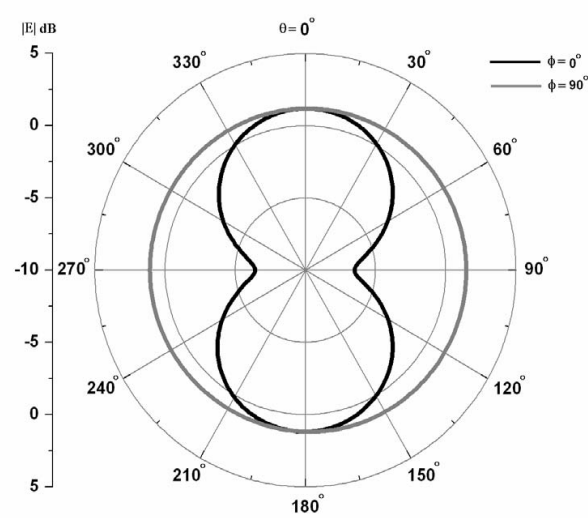

Fig. 7. Radiation Pattern of the U-shaped Antenna.

\section{RFID-enabled Sensor Nodes}

The capabilities of inkjet-printing technology in integrated wireless sensors on organic substrate bridging RFID and sensing technology was demonstrated in this section. The aim is to create a system that is capable of not only tracking provided by RFID protocol, but also monitoring. With this real-time cognition of the status of a certain object will be made possible by a simple function of a sensor integrated in the RFID tag. The ultimate goal is to create a wearable RFIDenabled sensor. For this effort, a microcontroller enabled UHF wireless sensor prototype has been developed.

The system level design for this wireless transmitter can be seen in Fig. 8 below. At the heart of the unit was an integrated 8-bit integrated microcontroller unit that was programmed to sample an analog temperature sensor, perform an analog to digital conversion of the sensed data, bit encode the digital form of the sensed data into full $2 \mathrm{sub}$ carrier cycle Miller bits and finally modulate the power amplifier in the integrated transmitter module in the same sequence as bit encoded, digital sensor data using Amplitude Shift Keying (ASK) modulation. The transmission frequency of $904.4 \mathrm{MHz}$ was generated by using a crystal oscillator that was connected to the input of the phase lock loop (PLL) of the transmitter. The entire wireless system including the antenna and the traces for the components mounting was printed on a 2-D module and operated remotely using a $3 \mathrm{~V} \mathrm{Li}$-ion cell.

The wireless sensor module is shown in Fig 9. U-shaped tapered RFID antenna was chosen in order to reduce the overall size while, especially that the traces for the sensors components were enclosed within the U-shape of the antenna while establishing a wide bandwidth near the center frequency that covers the UHF RFID frequency band. The Return Loss or S11 measurements for the center frequency for the antenna terminals was recorded to be $-15.05 \mathrm{~dB}$ for the simulated structure using the full wave EM simulator HFSS and $-12.45 \mathrm{~dB}$ measured using the ZVA- 8 VNA. The radiation pattern was also measured using Satimo's Stargate 64 Antenna Chamber measurement system and by using the NIST Calibrated SH8000 Horn Antenna as a calibration kit for the measured radiation pattern at $904 \mathrm{MHz}$. The radiation 
pattern of the antenna is quite similar to that of a classic dipole, which is desirable in most RFID applications.

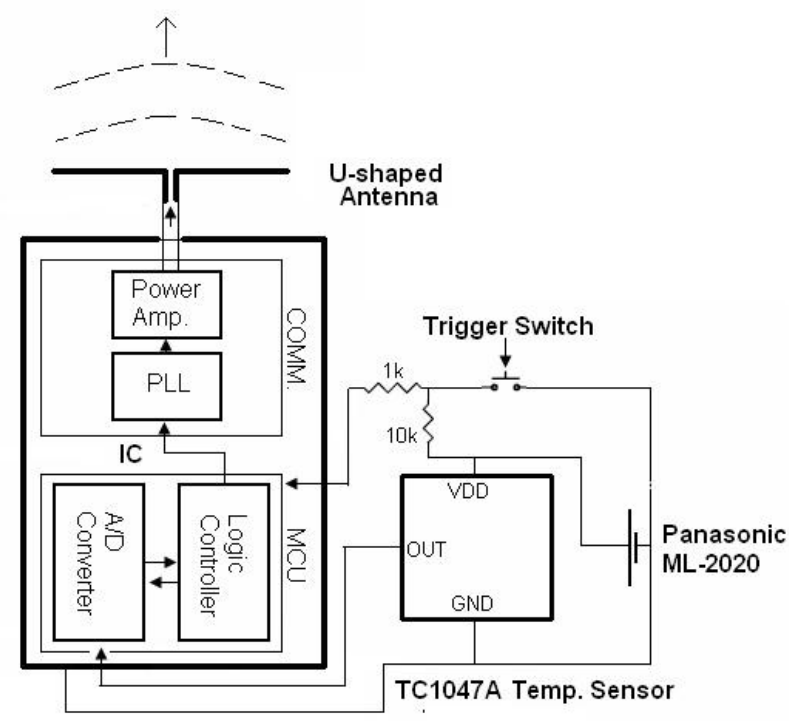

Fig. 8. System level diagram of wireless sensor module.

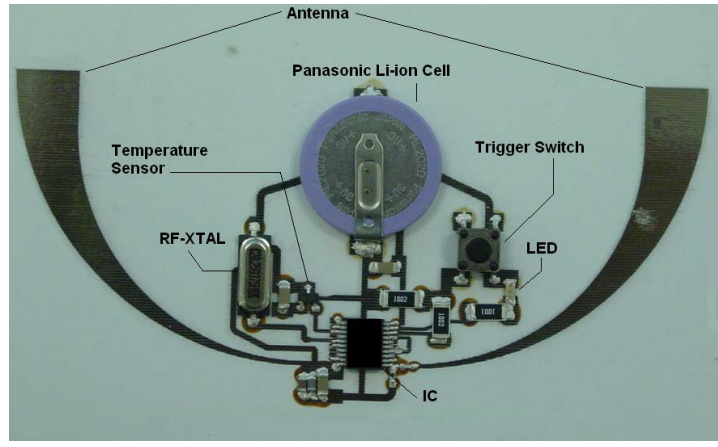

Fig. 9. Wireless Sensor transmitter prototype using silver inkjet printing technology.

\section{Conclusion}

The wearable RFID-enabled sensor nodes can find broad usage in real-time monitoring and medical monitoring applications. The capabilities of inkjet-printing technology ensure the low-cost fabrication by reel-to-reel mass process of textile. Key steps leading to a successful function module, such as dielectric characterization, RFID antenna design and integration of sensor were introduced in this paper. Preliminary embodiments show that the RFID-enabled sensor node could be effective for biomedical applications and wireless body area networks (WBAN).

\section{References}

[1] E. Jovanov, A. Milenkovic, C. Otto, P. D. Groen, B. Johnson, S. Warren, G. Taibi, "A WBAN System for Ambulatory Monitoring of Physical Activity and Health Status: Applications and Challenges," IEEE-EMBS 2005. 27th Annual International Conference, pp. 3810 - 3813, 2005.

[2] D. Thompson, O. Tantot, H. Jallageas, G. Ponchak, M. M. Tentzeris, J. Papapolymerou, "Characterization of LCP material and transmission lines on LCP substrates from 30 to $110 \mathrm{GHz}$," IEEE Trans. Microwave Theory and Tech., vol. 52, no. 4, pp. 1343-1352, April 2004.

[3] G. Zou, H. Gronqvist, J.P. Starski, and J. Liu, "Characterization of Liquid Crystal Polymer for High Frequency System-on-a-Package Applications," IEEE Trans. Advanced Packaging, vol. 25, no. 4, pp. 503-508, November 2002.

[4] A. Pique, and D. B. Chrisey, "Direct-write Technologies for Rapid Prototyping Applications", Academic Press, 2002. International Standard Book Number: 0-12$174231-8$ 